\title{
SOFTWARE LIVRE PARA BIBLIOTECAS, SUA IMPORTÂNCIA E UTILIZAÇÃO: O CASO GNUTECA ${ }^{1}$
}

\author{
Carlos Eduardo Navarro Ribeiro \\ Edilson Damasio
}

\section{Resumo}

Este artigo apresenta a importância da utilização de softwares para bibliotecas, tendo como foco o "software livre", suas vantagens e a utilização do Gnuteca-Sistema de Gestão de Acervo, Empréstimos e Colaboração para Bibliotecas, que foi desenvolvido pelo Centro Univ. Univates e está sendo utilizado no Brasil e em vários países. Utiliza a metodologia GNU e foi desenvolvido para utilizar os padrões MARC21 e importação de dados do Microisis. Apresenta os tipos de softwares, a importância dos softwares sem custos, e qual a definição de software livre. Tem como resultados de pesquisa bibliográfica a utilização do Gnuteca, suas vantagens para as bibliotecas e sua interoperabilidade com outros softwares, seus principais módulos e sua interface na Internet. Demonstrou-se que é um software viável às bibliotecas que não queiram ter custos com licenças de softwares.

\section{Palavras-chave}

Softwares para bibliotecas; Bibliotecas - informatização; Software livre

\section{FREE SOFTWARE FOR LIBRARIES, ITS IMPORTANCE And USE: THE GNUTECA CASE}

\begin{abstract}
This article presents the importance of the use of softwares for libraries, having "free software"point, its advantages and the use of "Gnuteca-Sistema de Gestão de Acervo, Empréstimos e Colaboração para Bibliotecas” (Gnuteca-System of Collection Management, Lending and Collaboration for Libraries), that was developed by the Center Univ. Univates, it is being used in Brazil and other countries. It uses the methodology GNU and was developed to use MARC21 standards and to import data of Microisis. The article presents the types of softwares, the importance of softwares without costs, and the definition of free software. It has as a result of bibliographical research, the use of Gnuteca, its advantages for the libraries, its interoperability with others softwares, its main modules and its interface in the InterNet. It was demonstrated that it is a viable software for libraries that do not want to have costs with licenses of softwares.
\end{abstract}

\section{Key words}

Softwares for libraries; Libraries - computerization; Free software

\footnotetext{
1 Trabalho apresentado no XXI Congresso Brasileiro de Biblioteconomia e Documentação, 2005, Curitiba.
} 


\section{INTRODUÇÃO}

Nos últimos anos tem-se observado o desenvolvimento muito rápido das tecnologias da informação assim como o desenvolvimento de softwares. Nesse meio, as bibliotecas e os profissionais que gerenciam informação têm-se deparado com uma grande quantidade de informações para organizar e também com uma grande quantidade de softwares.

Decidir quais tecnologias utilizar para o gerenciamento de informação não é uma decisão rápida como o seu desenvolvimento. Tem-se uma grande quantidade de opções, mas, todas as opções de softwares ou tecnologia da informação dependem principalmente de planejamento. O planejamento depende principalmente de recursos, tanto físicos, como humanos e financeiros.

Planejar qual software será necessário para uma biblioteca é um desafio aos bibliotecários, pois é uma tarefa difícil decidir, entre uma grande quantidade de opções, qual será a mais importante, tendo como critérios principais: o usuário da informação, os recursos de tecnologia da informação na instituição, os recursos financeiros, os humanos e o suporte de informática necessário. Estes critérios apresentados, já envolvem uma grande quantidade de tempo em planejamento e de preferência que siga o planejamento da instituição em que a biblioteca está inserida.

Conhecer os tipos de softwares disponíveis no mercado é uma tarefa simples, pois, catálogos de empresas com softwares proprietários são recebidos constantemente pelas instituições. Decidir por qual adquirir é considerado uma tarefa dos bibliotecários e equipe de planejamento, que gerenciam e administram as bibliotecas.

A ALA - American Library Association, indica, entre outros, as decisões em desenvolvimento de sistemas informatizados para bibliotecas, um quesito importante e disseminado na literatura, considerando que os bibliotecários têm a responsabilidade de aplicar a "eqüidade para o acesso à informação", tanto para os seus usuários como para a comunidade (ANCTIL, 2005). 
Decidir qual software adquirir é uma atividade difícil para todos os profissionais envolvidos. Desta forma, é necessário verificar o que o mercado tem a oferecer e os recursos disponíveis pela instituição.

Atualmente, a filosofia dos softwares livres tem sido muito disseminada e refere-se a softwares sem custos para aquisição, com desenvolvimento cooperativo e com os “códigos abertos”, ou seja, passíveis de adaptações e mudanças.

No presente artigo discute-se a utilização dos softwares, quais os tipos de softwares, quais são utilizados em Bibliotecas, qual a importância do "software livre”, e apresenta como estudo o software GNUTECA - Sistema de Gestão de Acervos, Empréstimos e Colaboração para Bibliotecas, destacando sua importância e sua utilização.

\section{SOFTWARES}

A cada dia cresce constantemente a necessidade de se ter um computador e a influência que os computadores têm sobre nossas atividades. O computador tem seu funcionamento auxiliado pelos programas que os conduzem em suas operações, esses programas, são denominados softwares. Um software é composto por instruções lógicas em linguagem de programação, ou seja, são algoritmos “uma seqüência finita de instruções cujo objetivo é solucionar um problema lógico ou matemático” (MICROSOFT, p. 124) escritos em forma a ser entendida e executada como instruções pelo computador, podendo, assim, futuramente, realizar os mais diversos tipos de tarefas.

De acordo com Sawaya (1999, p. 436) software é “suporte lógico, suporte de programação, um conjunto de programas, métodos e procedimentos, regras e documentação relacionados com o funcionamento e manejo de um sistema de dados”.

\section{TIPOS DE SOFTWARE}

No início da era da informática, por volta dos anos 50, a principal meta dos produtores de tecnologia de computadores era desenvolver técnicas e aperfeiçoamento de hardware, para a minimização de gastos e custos com armazenagem e organização de dados, além, é claro, 
da diminuição do tempo de processamento. Nessa época, o software era composto apenas de instruções lógicas para auxiliar o hardware na diminuição do tempo no processamento de cálculos.

Foi a partir dos anos 80 que se iniciou uma série de modificações nesse cenário. $O$ avanço tecnológico do momento passou a auxiliar ainda mais e o software passou a ser mais bem desenvolvido, porém, os desenvolvedores começaram a notar o mau aproveitamento dessa melhoria. Assim sendo, a solução encontrada para esse mal que se iniciava era finalmente o software (MEYER; BABER; PFAFFENBERGER, 2000).

A preocupação naquele momento voltava-se ao desenvolvimento e organização da produção de programas, que até então era básica e primaria, não deixando de lado a evolução da máquina, que continuava e continua até nossos dias em constante evolução.

Hoje, os dois segmentos, tanto o de hardware quanto o de software, continuam em constantes mudanças e desenvolvimentos. Os dois segmentos possuem amplo planejamento de produção e detecta-se a evolução contínua e paralela, ou seja, hardware evolui para acompanhar o software e vice e versa.

Vários tipos de softwares foram surgindo, Freeware, Shareware, Software Fechado, Livre, entre outros. Abaixo segue breve explicação sobre alguns deles:

Freeware: programas que geralmente são distribuídos gratuitamente. Alguns exigem cadastro para disponibilização, seu código fonte não é disponível, o que leva o software a não ser livre.

Shareware: de acordo com Microsoft Press (1998, p. 371) os sharewares possuem distribuição em caráter experimental e são protegidos por direitos autorais. Depois do período de experimento, normalmente, o usuário deve adquirir licença para dar continuidade a utilização. 
Software Fechado: são softwares que possuem seu código fonte fechado e são de propriedade privada, geralmente, através do Copyright. Esses softwares podem ser distribuídos gratuitamente, com autorização de quem mantém o Copyright.

Software Livre: é o software que pode ser utilizado, copiado, distribuído, aperfeiçoado, ou seja, modificado, por qualquer pessoa, mesmo não sendo proprietária. A seguir estudaremos mais precisamente um software livre.

\section{SOFTWARE LIVRE}

Atualmente está ocorrendo uma crescente evolução no mundo dos softwares. Diariamente os desenvolvedores, ou seja, programadores, buscam inovações e técnicas para acompanhar os melhoramentos na tecnologia de hardwares e periféricos. Perante essa realidade, surge o propósito da utilização de tecnologia alternativa. Assim sendo, torna-se claro que o mundo caminha para a órbita dos softwares livres, em busca da possibilidade de adaptação legal.

Um programador, desenvolvedor e ou construtor de softwares, utiliza como ferramenta básica para o desenvolvimento e aperfeiçoamento de seus produtos o código fonte de um sistema. Um software livre é aquele que possui seu código fonte aberto a qualquer usuário, que queira ou necessite de modificações e adaptações, seja para uso doméstico, institucional ou empresarial.

Os programas com código fechado, são licenciados através do copyright. Por outro lado, o copyleft licencia os softwares com código fonte aberto:

O copyleft é o nome, um trocadilho com copyright, designado às licenças de propriedade intelectual (nome genérico que abrange os direitos autorais, de software e de marcas) que permitem a qualquer pessoa usar, comercializar, copiar, ter acesso a todas as informações e modificá-las, desde que na versão modificada, pelo menos na parte em que o original foi utilizado, também permita essas mesmas liberdades (PEREIRA, 2004). 
As primeiras idéias sobre desenvolvimento de software livre foram criadas por Richard Stallman, no fim da década de 70. Foi Stallman que deu início à Fundação de Software Livre (Free Software Fundation). De acordo com MICROSOFT (1998, p. 371) criada para eliminar restrições sobre os direitos de uso, distribuição, cópia e modificação de programas para computador, possuindo código fonte aberto. A Free Software Fundation mantém e distribui softwares do tipo GNU (FREE SOFTWARE FUNDADION, 2005).

\section{GNU}

O GNU teve o início de sua filosofia por volta de 1984 e tinha como objetivo principal, desenvolver um sistema operacional completo, porém, livre na tentativa de ser compatível ao UNIX ${ }^{1}$. De acordo com Sawaya (1999, p. 203) “GNU, acrônimo para “GNU's not Unix” (“Gnu não é Unix”) é um projeto cujo objetivo é fornecer, gratuitamente, software compatível Unix.”

Os GNU/Linux são sistemas operacionais livres, compatíveis com o Unix. Eles são mantidos por programadores do mundo todo, interligados pela Internet, com objetivo de sempre criar melhorias.

O GNU/Linux, proporciona ao usuário estabilidade no funcionamento do computador além de manter baixo custo e ótimo desempenho em redes de computadores.

\section{SOFTWARES PARA BIBIOTECAS}

Os softwares para bibliotecas tiveram seu início através da inserção da informática na sociedade, acompanhando sempre seu desenvolvimento e as novas tecnologias da informatização. Primeiramente com softwares que emitiam listagens em forma de referências, ou fichas catalográficas, como o D-BASE, para serem utilizadas nos catálogos de fichas das bibliotecas. Destaca-se também que nas bibliotecas de grande porte, na década de 70 eram utilizavam os computadores Mainframe da IBM.

Os softwares desenvolvidos para aplicações em computadores de grande porte, se, por um lado, possuíam grande capacidade de armazenamento de dados, por outro, não permitiam a 
alimentação em tempo real e exigiam infra-estrutura computacional com equipes altamente especializadas e ambientes totalmente apropriados, colocando as bibliotecas, os bibliotecários e os usuários totalmente dependentes da tecnologia com pouca agilidade na prestação de serviços. A informação sucumbe-se à tecnologia (CÔRTE et al., 1999, p. 242).

Após a utilização das tecnologias dos PCs (Personal Computers) e o desenvolvimento das Tecnologias da Informação, estes modelos de softwares foram sendo atualizados e desenvolvidos para uma parcela do mercado que não tinha acesso a computadores de grande porte. Acompanhando sempre o desenvolvimento da Tecnologia da Informação e o desenvolvimento dos Sistemas Operacionais.

A modernização das bibliotecas está diretamente ligada à automação de rotinas e serviços, com o intuito de implantar uma infra-estrutura de comunicação para agilizar e ampliar o acesso à informação pelo usuário, tornando-se necessário haver uma ampla visão da tecnologia da informação e sua aplicação nas organizações (CÔRTE et al., 1999, p. 242)

Neste contexto surgiram as empresas de desenvolvimento de softwares.

Os softwares para bibliotecas surgiram através de diversas implementações e desenvolvimentos, aliados aos recursos de Tecnologia da Informação existentes nas Instituições de ensino e empresas. Desenvolveram-se inúmeros softwares, utilizando os requisitos mínimos de informações sobre catalogação, a organização de cabeçalhos de assuntos e a padronização automática de índices, tendo como padrão principal de formatação dos dados, o formato MARC, desenvolvido e disseminado pela U. S. Library of Congress - Biblioteca do Senado Americano.

As bibliotecas começaram a implementar bases de dados também nos formatos padronizados e utilizados para a catalogação, conforme os padrões adotados internacionalmente, como o ISBD e AACR2, este último utilizado no Brasil. Tendo seus dados organizados para estes padrões e o formato de importação de dados MARC, as bases de dados começaram a se interligar e a ter um padrão mínimo para a área biblioteconômica. 
No final da década de 80 surgiu o MicroIsis - CDS-ISIS, software desenvolvido pela Unesco e que hoje é um dos softwares mais popularizados para bibliotecas, no Brasil e no mundo, devido a sua licença Freeware, não sendo necessário o pagamento de licenças.

Dentre os softwares mais utilizados, destaca-se o CDS-ISIS, modelo de softwares para o gerenciamento de documentação através de bases de dados de fácil programação, sendo acessível e programável por profissionais bibliotecários e da área de informática. Foi implementado e distribuído mundialmente pela UNESCO ao mundo todo. Teve sua primeira versão na década de 80, distribuída na América Latina.

O Microisis tem como principal função organizar os dados dos materiais catalogados e distribuí-los em bases de dados.

O Microisis (CDS-ISIS) foi utilizado, inicialmente, na versão DOS e até o momento é distribuído na forma de Freeware, com os códigos fechados mas sem custos para a utilização de sua licença, sendo necessário somente um cadastro de usuários.

Uma de suas facilidades é a programação e implementação de relatórios documentais em diversos formatos, podendo ser programável pelos próprios usuários ou suporte de informática.

A característica principal do Microisis é sua função de base de dados central, tendo opções para a inserção de dados multiusuário, através de sua implementação em rede e opção para consultas a catálogos nas bibliotecas, através do desenvolvimento de módulos de catálogo.

Diversos softwares com licenças para compra, softwares fechados, foram também implementados para utilizar os dados cadastrados no Microisis. Estes softwares complementam com módulos de serviços que não possui implementação. Eles utilizam uma interface para a utilização dos dados para outras funções, como catálogos, circulação, relatórios estatísticos, e finalmente ao catálogo via Internet. Utilizam um sistema que faz a ligação entre a base de dados e os outros módulos, hoje denominado ISISDLL, desenvolvido em conjunto BIREME - Centro Latino-Americano e do Caribe de 
Informação em Ciências da Saúde e UNESCO, para sua versão em DOS ou Windows. “[...] o ISIS_DLL é uma interface de aplicação aplicada, para o desenvolvimento de softwares utilizando a tecnologia CDS/ISIS. A DLL (Dynamic Link Library) é independente da linguagem de programação usada, utilizado para hospedar o aplicativo”. (UNESCO, 2005, tradução dos autores).

Desta forma, outros sistemas podem e são desenvolvidos com o objetivo de ler os dados denominados "bibliotecas" de outros sistemas, ou seja, por outras formas de programação gráfica, como: Windows escrita em Visual Basic, Delphi, C, C++, Pascal.

Com o ISISDLL a versão em Windows, denominada WINISIS, facilitou muito o desenvolvimento de módulos, que faltavam no Microisis, através de programação em outras linguagens.

Neste quesito a Bireme, organismo internacional, desenvolveu diversos produtos para a implementação de novos módulos ao Microsis e a distribuição de licenças de outros programas, que possibilitam melhorias na sua utilização, dentre eles destacam-se:

SeCS - Sistema que permite o registro e acompanhamento de coleções de publicações seriadas.

EMP - Sistema para gerenciamento de serviços de empréstimos de bibliotecas.

WWWISIS - é o servidor de Internet para bases de dados Isis. Opera via CGI (Commmon Gateway Interface) do Hypertext Transport Protocol (HTTP) e, a partir da versão 4.0, conhecida também como WXIS, utiliza uma linguagem de script baseada em XML ${ }^{2}$, o IsisScript (BIREME, 2005).

Mesmo com este desenvolvimento de softwares Freeware dos módulos de empresas particulares para aquisição, também foram desenvolvidos diversos outros softwares comerciais. Com as mesmas funções e nível de desenvolvimento em forma de competição de mercado, sendo melhorados constantemente. No mercado internacional e nacional existem softwares e sistemas para Bibliotecas, alguns com implementações e módulos além das expectativas das bibliotecas e dos profissionais bibliotecários, e também além dos recursos financeiros da maioria das bibliotecas do mundo, desta forma substituindo o 
Microisis e outros softwares Freeware, com implementações necessárias também às bibliotecas.

Existe também a possibilidade das instituições ou empresas adquirirem estes softwares e suas licenças Freeware, como o Microisis, mas existem vantagens e desvantagens, tanto operacionais e principalmente financeiras.

\section{SOFTWARE LIVRE PARA BIBLIOTECAS}

Os Princípios de Keystone, Clarke² (2000) apud (ANCTIL; BEHESHTI, 2005) apresentam a importância do bibliotecário estipular qual software deva utilizar, através do princípio da eqüidade de acesso à informação.

No Brasil foi desenvolvido o software GNUTECA - Sistema de Gestão de Acervo, Empréstimo e Colaboração para Bibliotecas, primeiramente instalado e desenvolvido na UNIVATES, conforme abaixo:

O Gnuteca é um sistema para automação de todos os processos de uma biblioteca, independentemente do tamanho de seu acervo ou da quantidade de usuários. O sistema foi criado de acordo com critérios definidos avalidados por um grupo de bibliotecários e foi desenvolvido a partir de testes em uma biblioteca real, a do Centro Universitário Univates, onde está em operação desde fevereiro de 2002. O Gnuteca é um software livre, o que significa que o mesmo pode ser copiado, distribuído e modificado livremente. O software é aderente a padrões conhecidos e utilizados por muitas bibliotecas, como o MARC21 (LOC - Library Of Congress). Por ter sido desenvolvido dentro de um ambiente CDS/ISIS, o Gnuteca prevê a fácil migração de acervos deste tipo, além de vários outros (GNUTECA, 2005).

${ }^{2}$ CLARKE, Kevin S. Open software and the library community. 2000. Thesis (Master's)-School of Information and Library Science, University of North Carolina, 2000. 
O Gnuteca também é distribuído tendo como princípio a cooperação para o seu desenvolvimento entre os seus usuários. Quando desenvolve-se novos módulos, pode-se implementá-los em novas versões do software - no momento está na versão 1.5.

A questão do não custo às instituições, bibliotecas ou usuários é o que possibilita a implantação e uso dos softwares livres.

A escolha pelo desenvolvimento de um sistema complexo em software livre é viável quando comparada à aquisição de um produto proprietário equivalente, mesmo quando apenas uma instituição arca com este custo. Quanto maior o número de instituições envolvidas na produção de sistemas que possam ser compartilhados, menor o custo total de desenvolvimento e suporte, e maior a velocidade de produção (com mais pessoas envolvidas) (SALVI et al., 2005).

O Gnuteca tem o protocolo GNU Linux ou Windows, e, portanto, pode ser utilizado nos sistemas operacionais mais utilizados no mundo (GNUTECA, 2005).

Salvi et al. (2005) esclarece:

É um sistema abrangente e genérico que pode moldar-se a diferentes realidades de diferentes usuários, permitindo também a criação de uma infra- estrutura de colaboração entre bibliotecários e demais funcionários das bibliotecas, evitando a repetição desnecessária de trabalho: uma vez feita a catalogação de um título em uma biblioteca, estes dados catalográficos podem ser "importados" para o sistema de outra biblioteca que adquira o mesmo título.

Na área de biblioteconomia, os principais vantagens são:

- custo zero na aquisição de licenças;

- catalogação em formato MARC21;

- gerenciamento do processo de circulação;

- várias opções de busca ao catálogo local ou on-line; 
- várias opções de serviços aos usuários;

- renovações de empréstimos via Internet;

- $\quad$ reserva de obras via Internet;

- catalogação multiusuário;

- possibilidade de integração de Sistemas de Bibliotecas, ou Câmpus e Bibliotecas Setoriais e Central (Sistema Integrado de Bibliotecas);

- catalogação cooperativa através da troca de dados catalogados;

- opções de migração dos dados do Microisis, dados no formato ISO 2709;

De acordo com Weisheimer (2004) o Gnuteca tem os seguintes módulos e suas funções principais:

Módulo de pesquisa:

- pesquisa simples; pesquisa multicampo; percorrer índices

- pesquisa simples com a opção de Bibliotecas e campos (todos, autor, título, entre outros)

- pesquisa multicampo com a opção de Bibliotecas e 2 caixas de texto para consulta, com opções multicampo e utilização de operadores booleanos.

Módulo administração:

- $\quad$ acessos e permissões a membros da equipe da Biblioteca.

- $\quad$ inserir e administrar: 
- unidades da biblioteca; grupos de usuários; estados dos exemplares; situação da reserva; políticas dos grupos; feriados; gêneros de materiais; direitos dos grupos de usuários; multas; empréstimos; reservas; usuários; entre outros.

Modulo de Empréstimos:

- empréstimos por nome; pendentes; alterar por tombo; listas atrasados; listar multas; histórico.

Modulo de Reservas

- Não atendidos; atendidos; por tombo; atualização.

Módulo de Usuários

- vínculos; pessoas.

Módulo de importação de arquivos no formato ISO 2709, denominado ImportaIso e desenvolvido para migrar dados do CDS-ISIS ao Gnuteca.

Estas seriam algumas das funcionalidades do Gnuteca e sua versão está disponível para download, livremente em sua página na Internet http://www.gnuteca.org.br .

\section{CONCLUSÃO}

Softwares para bibliotecas devem ser utilizados na era atual como um complemento dos serviços bibliotecários, e principalmente por todas as bibliotecas que tenham recursos na área de Tecnologia da Informação. A opção de como adquirir e onde adquirir, fica a critério de cada biblioteca e seus recursos disponíveis, tanto físicos, técnicos e principalmente financeiros. 
A filosofia de softwares livres, em relação às bibliotecas, foi instituída pela Unesco com o desenvolvimento e disseminação do Microisis. Muitas instituições organizaram e ainda organizam seus acervos informacionais com este software. Por ele ser totalmente adaptável, no quesito relatórios e, principalmente, acessível sem custos.

A filosofia dos softwares livres é elogiada e criticada por pensadores, pesquisadores e críticos, discutida na política de aquisição de softwares pelos órgãos públicos, principalmente das esferas federal e estaduais, e também por instituições que não queiram adquirir licenças de softwares proprietários.

Os softwares livres são uma opção para instituições ou pessoas físicas que não queiram ter gastos na aquisição destes. Referem-se não somente a softwares para bibliotecas. mas também sistemas operacionais como o Linux e diversos softwares como o OpenOffice, por exemplo.

Existem diversos softwares livres para bibliotecas sendo utilizados, desenvolvidos e disseminados no mundo todo. O software Gnuteca tem a importância de ser um software desenvolvido de forma cooperativa, sendo que já teve várias versões desenvolvidas e constantemente atualizadas e é utilizado por várias instituições no Brasil e no mundo. Sua interoperabilidade com outros softwares é também uma de suas vantagens, pois, com o código aberto, permite adaptações a outros softwares institucionais também livres. Desta forma, possibilita às instituições terem um software sem custos para a biblioteca, e a instalação de outros softwares que interajam com ele, como, por exemplo, sistemas operacionais, sistemas financeiros, controles acadêmicos, softwares de gerenciamento de bases de dados, geração de relatórios, entre outros.

Outra vantagem do Gnuteca é que ele utiliza o padrão MARC21 para a inserção dos dados no sistema e foi desenvolvido com a assessoria de bibliotecários. Oferece recursos de configuração, catalogação, administração, pesquisas e serviços aos usuários, com interface de interatividade fácil para os usuários, bibliotecários, auxiliares, técnicos de bibliotecas e suporte de informática. Além de disponibilizar sua interface de consulta e serviços na Internet. Desta forma, não há fronteiras ou dificuldades de comunicação entre usuários e biblioteca, ou bibliotecas com outras bibliotecas. 
Outra facilidade do Gnuteca foi ser desenvolvido no sentido de permitir importar dados do Microisis, tendo um módulo para a importação de arquivos ISO 2709, padrão utilizado pelo Microisis e outros softwares de documentação.

Desta forma o Gnuteca é uma interessante opção para as instituições, bibliotecas ou bibliotecários que queiram organizar acervos informacionais ou queiram realizar mudança de sistemas em suas bibliotecas.

\footnotetext{
${ }^{1}$ Sistema operacional considerado poderoso, multiusuário e multitarefa, produzido por Ken Thompson e Dennis Ritchie no Bell Laboratóries.

${ }^{2}$ Extensive Markup Language. Linguagem de Internet com o texto marcado, possibilitando sua organização e recuperação dos dados.
}

\section{REFERÊNCIAS}

ANCTIL, E.; BEHESHTI, J. Open source integrated Library Systems: an owerview. Disponível em: < http://www.anctil.org/users/eric/oss4ils.html >. Acesso em: 20 jan. 2005.

BIREME. Catálogos de produtos. Disponível em:

< http://productos.bvsalud.org/html/pt/home.html >. Acesso em: 07 jan. 2005.

CÔRTE, A. R.; et al. Automação de bibliotecas e centros de documentação: o processo de avaliação e seleção de softwares. Ciência da Informação, Brasília, DF, v. 28, n. 3, p. 241256, set./dez. 1999.

FERIA, L.; ROSAS BARAJAS, J. R. A tres años del 2000: la situación de la información electrónica en los países latinoamericanos. In: JORNADAS MEXICANAS DE BIBLIOTECONOMIA, 1996. Anais... Colima: Universidad de Colima, 1996.

FREE SOFTWARE FUNDATION-FSF. Disponível em: < http://www.fsf.org/ > . Acesso em: 20 jan. 2005.

GATES, B. A estrada do futuro. São Paulo: Companhia das Letras, 1995.

GNUTECA - Sistema de Gestão de Acervo, Empréstimo e Colaboração para Bibliotecas. Disponível em: < http://www.gnuteca.org.br >. Acesso em: 20 mar. 2005.

MEYER, M.; BABER, R.; PFAFFENBERGER, B. Nosso futuro e o computador. 3. ed. Porto Alegre: Bookman, 2000.

MICROSOFT PRESS dicionário de informática. Rio de Janeiro: Campus, 1998.

PEREIRA, A. L. G. Copyright x Copyleft: uma análise marxista da indústria de software. Cadernos do SepAdm, Salvador, n.1, 2004. Disponível em:

http://www.adm.ufba.br/copyright_copyleft-uma_analise.pdf>. Acesso em 30 jan. 2005. 
SALVI, E. J.; et al. Quando o bazar não funciona: custo total de "propriedade" no desenvolvimento de um sistema complexo em software livre, o GNUTECA. Disponível em: < http://www.varianet.com.br/articles/gnuteca.pdf > . Acesso em: 20 mar. 2005.

SAWAYA, M. R. Dicionário de informática e internet: inglês - português. São Paulo: Nobel: CEETEPS, 1999.

UNESCO. ISIS_DLL the programming interface for developing CDS/ISIS software. Disponível em: < http://portal.unesco.org/ci/en/ev.phpURL_ID=5333\&URL_DO=DO_TOPIC\&URL_SECTION=201.html >. Acesso em: 20 jan. 2005. 


\section{Edilson Damasio}

Bibliotecário.

Mestre em Ciência da Informação.

Editor da Revista Maringá Management: Revista de Ciências Empresariais.

edamasio@uem.br

\section{Carlos Eduardo N. Ribeiro}

Graduando em Administração em Análise de Sistemas.

Auxiliar de Biblioteca. Centro de Ensino Superior do Paraná - CESPAR - Biblioteca Central carlos@faculdadesmaringa.br

Recebido em: 20/03/2006

Aceito para publicação em: jun. 2006 